\title{
STUDY OF TEMPORAL VARIATIONS OF ELECTRICAL RESISTIVITY \\ AND ELECTRIC FIELDS ON THE SAN ANDREAS FAULT
}

H.F. Morrison and R. Fernandez

USGS CONTRACT NO. 14-08-0001-18390

Supported by the EARTHQUAKE HAZARDS REDUCTION PROGRAM

OPEN-FILE NO.81-379

U.S. Geological Survey

OPEN FILE REPORT

This report was prepared under contract to the U.S. Geological Survey and has not been reviewed for conformity with USGS editorial standards and stratigraphic nomenclature. Opinions and conclusions expressed herein do not necessarily represent those of the USGS. Any use of trade names is for descriptive purposes only and does not imply endorsement by the USGS. 
The objective of this program is to develop electrical and electromagnetic systems for geothermal exploration. With the development in LBL of very successful de resistivity, electromagnetic (EM60) and remote reference magnetotelluric systems, the emphasis is currently shifting to improved experimental design and interpretation.

A major effort has also been devoted to improved data acquisition and processing. A new in-field processing system has been successfully tested. This system includes a Hewlett-Packard desk top computer, Nicolet Explorer III Digital Oscilloscope and an analog multiplexer of our design. A field test of a prototype system conducted in Nevada in the summer of 1980 was quite encouraging. This system can also interface the existing digital signal processor to expand its capability, including power spectral averaging, in-field graphics, gain and filter control and in-field modelling.

Other major activities are isited below. Highlights of the program follow in expanded description.

i. Fundmental studies of electromagnetic response of the ground for magnetic dipole sources.

ii. Improved high speed and cost effective numerical modelling algorithms.

iii. Studies of direct inversion schemes for two-dimensional models.

iv. Study of topographic effects in magnetotellurics.

v. Field experiments with the new remote reference noise cancellation scheme for EM60. 
A number of reports on electrical resistivity variations associated with earthquakes has stimulated further research into this promising earthquake prediction tool. After several years of failing to detect such precursors before strike-slip earthquakes in the area, it was felt necessary to examine carefully the temporal variations (i.e. noise) in the apparent resistivity and to seek ways to improve the measurement accuracy.

To improve the measurement accuracy, new instrumentation has been developed. Rigorous laboratory and field tests led to further modifications in order to increase reliability and accuracy.

A principal result of this study has been the identification of the major sources of noise. It has been found that large geomagnetically induced telluric currents are responsible for errors during unusual periods of high geomagnetic activity. Preliminary tests using a remote electric field measuring site to cancel the locally induced telluric noise have been conducted. When this activity is low, it is necessary to increase the gain at the HERMIT receivers in orcier to have a resonable noise voltage amplitude and be able to apply this scheme successfully.

Unsuccessful results were obtained when we measured the remote magnetic fields. As a result, the transfer function between the local and remote site became very unstable as a function of time.

Resistivity variations have been measured repeatedly to accuracies of $\pm 0.1 \%$ for selective sites over a period of more than a year and are shown in Figure 1. In this period we have observed resistivity variations which take place from week to week and amount to a fraction of $1 \%$, and in some cases even more than $1 \%$. After a detailed statistical 
analysis of these data, we have found strong indications that these changes are the result of surface resistivity variations. Resistivity changes ocurring at a local electric dipole near the transmitter (EMM 2-3) correlate very well with resitivity changes taking place at the rest of the sites. These sites however, are located at distances greater than $7 \mathrm{Km}$ from the transmitter. After a careful consideration of other factors that might be responsible for these correlations, it was determined that the cause of these correlations can most likely be attributed to a current channeling phenomena that takes place throughout the highly conductive fault zone.

The current channeling phenomena can be considered one of the most important results of this study. It should be clear however, that to be able to determine the existence of such phenomena it was necessary to reduce the error in the measurement to the levels here achieved. That in itself was not easy.

A study of this kind lends itself to a large number of suggestions for further research. It is hoped that some of these suggestions can be selected from some of the positive and negative results obtained here. If electrical resistivity monitoring is going to be used in search for possible recursors of seismic activity, it is essential to study in detail the particular area where these measurements are going to take place. In the Hollister area we showed that, unless there are means by which one could place current and measuring electrodes to avoid the first 100 to $200 \mathrm{~m}$ of highly conductive material (believed to be responsible for current channeling effects) further monitoring of apparent resitivity may not provide the desired results. 


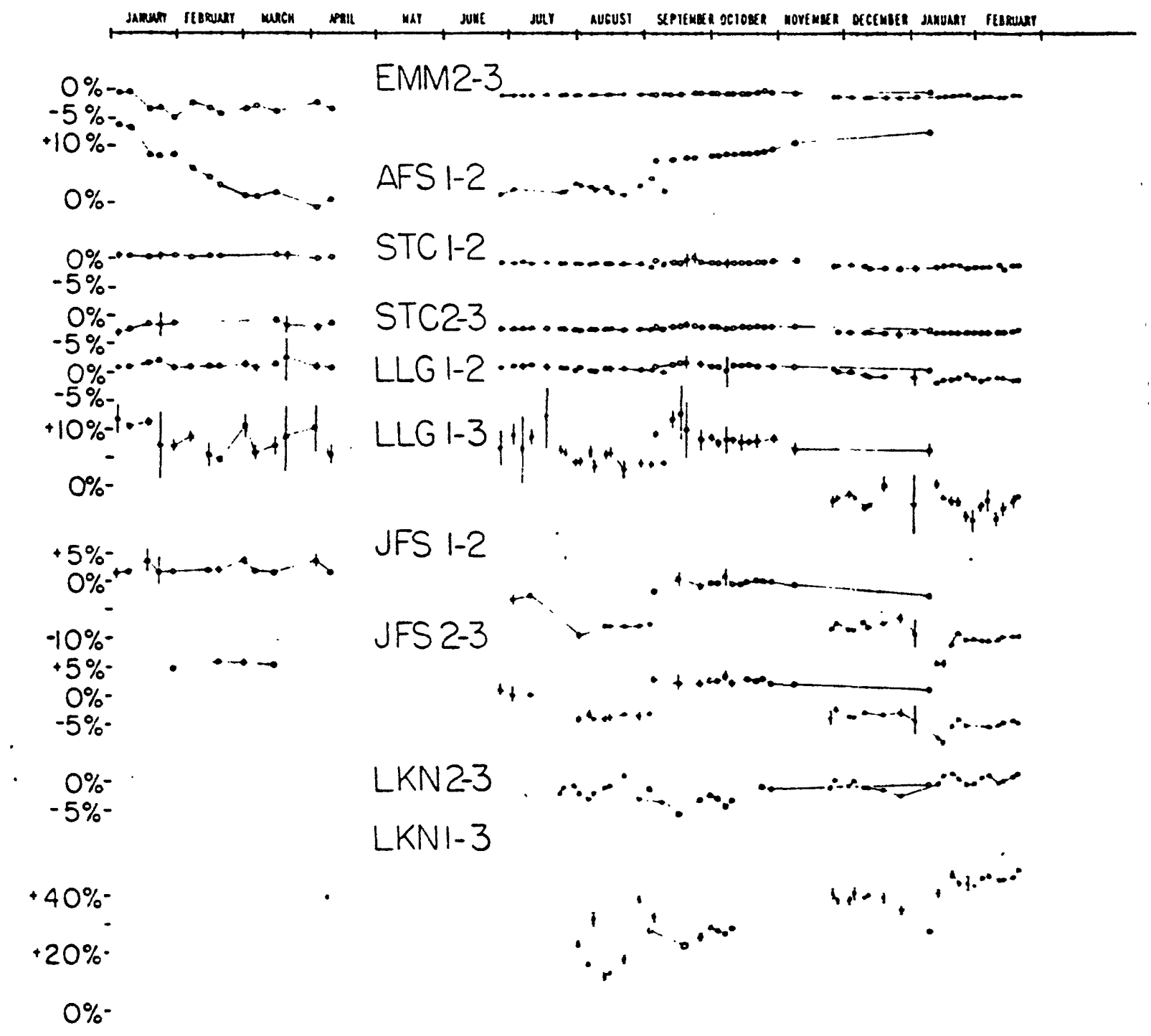

Figure 1: Resistivity data for 1979 and 1980. Open circles correspond to data obtained with a gasoline-powered transmitter full circles for data obtained with a line-powered transmitter. 